\title{
A novel approach to multi-criteria inverse planning for IMRT
}

\author{
Sebastiaan Breedveld ${ }^{1}$, Pascal R M Storchi ${ }^{1}$, Marleen Keijzer ${ }^{2}$, \\ Arnold W Heemink ${ }^{2}$ and Ben J M Heijmen ${ }^{1}$ \\ ${ }^{1}$ Department of Radiation Oncology, Erasmus MC Rotterdam, Groene Hilledijk 301, \\ 3075 EA Rotterdam, The Netherlands \\ 2 Delft University of Technology, Delft Institute of Applied Mathematics, PO Box 5031, \\ 2600 GA Delft, The Netherlands \\ E-mail: s.breedveld@erasmusmc.nl
}

Received 4 July 2007, in final form 6 September 2007

Published 2 October 2007

Online at stacks.iop.org/PMB/52/6339

\begin{abstract}
Treatment plan optimization is a multi-criteria process. Optimizing solely on one objective or on a sum of a priori weighted objectives may result in inferior treatment plans. Manually adjusting weights or constraints in a trial and error procedure is time consuming. In this paper we introduce a novel multi-criteria optimization approach to automatically optimize treatment constraints (dosevolume and maximum-dose). The algorithm tries to meet these constraints as well as possible, but in the case of conflicts it relaxes lower priority constraints so that higher priority constraints can be met. Afterwards, all constraints are tightened, starting with the highest priority constraints. Applied constraint priority lists can be used as class solutions for patients with similar tumour types. The presented algorithm does iteratively apply an underlying algorithm for beam profile optimization, based on a quadratic objective function with voxeldependent importance factors. These voxel-dependent importance factors are automatically adjusted to reduce dose-volume and maximum-dose constraint violations.
\end{abstract}

(Some figures in this article are in colour only in the electronic version)

\section{Introduction}

Traditionally, irradiating the tumour with the prescribed dose has been the primary goal in radiotherapy, as long as no critical damage is done to the OARs. Nowadays, with better planning and treatment tools available, optimal sparing of OARs has also become a major goal. One of the difficulties in plan optimization is that feasible solutions may heavily, and rather unpredictably, depend on the selected objectives and constraints, and their relative weights. In clinical practice, dosimetrists do usually optimize constraints, objectives, and/or 
weights in a time-consuming interactive trial-and-error process, to find some acceptable compromise. Often constraints are implemented as objectives and optimized in a weighted sum function, making it impossible to make a sharp trade-off between objectives without violating constraints. Multi-criteria optimization (or multi-objective optimization) aims at providing tools to steer this process. One approach includes the generation of sets of Pareto efficient solutions (Cotrutz et al 2001, Lahanas et al 2003, Küfer et al 2005, Craft et al 2005, 2006, Halabi et al 2006, Hoffmann et al 2006).

In this paper, a novel approach to multi-criteria optimization is presented. This algorithm maximizes or minimizes objectives subjected to constraints. An objective is implemented as a soft constraint, a constraint which is allowed to be violated and adapted by the algorithm. (To distinguish normal constraints from soft constraints, constraints which are not allowed to be violated are called hard constraints.) The objectives are selected and prioritized a priori by the radiation oncologist and grouped together with the (hard) constraints to a constraint priority list. Soft constraints with a low priority may be automatically relaxed to meet higher priority or hard constraints (e.g. the volume allowed to receive more than the critical dose is increased for a dose-volume constraint). Soft constraints may also be tightened where possible. Finally, for each patient a single plan is generated with a set of constraints that just avoids constraint violations; tightening any of the final constraints will result in at least one violation for the other constraints. The final plan meets all hard constraints and the soft constraints are met as well as possible.

The proposed algorithm for multi-criteria optimization iteratively applies an in-house developed algorithm for beam profile optimization. With the latter algorithm, profiles are optimized using voxel-dependent importance factors in a quadratic dose objective function. Optimization includes automatic adjustment of these factors in order to reduce dose-volume and maximum-dose constraint violations. An algorithm for fast minimization of quadratic functions has recently been published (Breedveld et al 2006), and is applied here. Despite that the multi-criteria optimization algorithm is only applied to our in-house developed beam profile optimization, we believe that the algorithm can be applied to a broader range of algorithms for constrained optimization, including, for example, aperture based optimization.

To weigh the relative importance of OARs and the tumour in inverse planning for IMRT, a volume-wide importance factor can be used for each volume (Bortfeld et al 1990, Brahme 1995, Spirou and Chui 1998, Wu and Mohan 2000). Because the desired dose is more easily delivered to some voxels than to others, the volume-wide importance factor can be refined to voxel-dependent importance factors. This provides more local control over the dose (Cotrutz and Xing 2002, Wu et al 2003). The weights of the voxels can be chosen in advance by looking at the depth and position of the organs (Shou et al 2005) or adapted in an iterative procedure (Cotrutz and Xing 2003, Yang and Xing 2004).

Recently, Wilkens et al (2007) and Jee et al (2007) have also studied the use of constraint priority lists, using different optimization schemes and different underlying optimization algorithms. These papers also describe the advantage of goal programming/lexicographic ordering in treatment planning. Wilkens et al formulate a four-step approach which is used as a class solution for treating head and neck patients. The fourth step incorporates a non-clinical goal: smoothing of the fluence. In our approach, profile smoothing is an integral part of the optimization procedure, accounted for by a dedicated term in the objective function. Jee et al describe the application of lexicographic ordering with four levels of priority, applied to a prostate and a head and neck case. For the prostate case they also show the impact of changing the priorities of PTV irradiation and maximum dose to the rectum wall.

One of the main differences between the approaches of Wilkens et al and Jee et al and our approach is that their objectives are handled one by one, and in a pre-defined order. Our 
approach first tries to find a solution, fulfilling all constraints. If that is not possible, limiting (lower-priority) constraints are relaxed. However, after relaxation, the primary goal of the algorithm becomes meeting all initial constraints (as prescribed by the radiation oncologist). This process starts with the highest priority constraints that have been relaxed. If, for example, the dose in the parotids is within the constraints, it may be more important to first lower the dose in the oral cavity. If all the initial constraints are met (or as well as possible), an attempt is made to tighten constraints below the initially prescribed levels, again starting with the highest priority constraints.

\section{Methods and materials}

\subsection{Global description of optimization routines}

The proposed multi-criteria approach is based on algorithms for optimization of (1) beam profiles, (2) voxel weights and (3) imposed treatment constraints. To define the problem, tumour dose prescriptions, dose-volume and maximum-dose constraints, a constraint priority list, and volume-wide importance factors have to be set. This defines the planning protocol. Ideally, a single protocol can be applied to patients with similar tumour types.

For the beam profile optimization a quadratic objective function is used to minimize the difference between the desired or prescribed doses in voxels and the attained doses. Each voxel has a coefficient $\eta$. The higher the coefficient, the more likely it becomes for that voxel to meet its ideal dose objective, either 0 Gy dose for organs at risk, or the prescribed dose for the tumour. (2006):

The quadratic objective function used has been discussed thoroughly in Breedveld et al

$$
s(\boldsymbol{f})=\sum_{v \in \mathcal{V}} \xi_{v}\left(H \boldsymbol{f}-\boldsymbol{d}_{v}^{p}\right)^{T} \tilde{\eta}_{v}\left(H \boldsymbol{f}-\boldsymbol{d}_{v}^{p}\right)+\kappa(M \boldsymbol{f})^{T}(M \boldsymbol{f})
$$

The first term is the quadratic dose objective, modified for use with voxel-dependent importance factors. $H \boldsymbol{f}$ is the dose resulting from the fluence $\boldsymbol{f}$, and $\boldsymbol{d}_{v}^{p}$ is the dose objective for voxels in volume $v$ (from the set of all volumes $\mathcal{V}$ ). The volume-wide importance factors $\xi_{v}$ are still used as a priori weighting between volumes. The vector of voxel-dependent importance factors $\boldsymbol{\eta}_{v}$ (which will be abbreviated as the voxel-coefficient vector $\boldsymbol{\eta}$ for readability) is written as a diagonal matrix $\tilde{\eta}_{v}$. In this approach, the dimension of the coefficient vector equals the number of patient voxels. Only the subset of the coefficients corresponding to the volume $v$ can be unequal to zero. Because each volume has its own voxel-coefficient vector, one voxel (in the patient space) can belong to more than one volume (i.e. volumes can overlap). The second term $\kappa(M f)^{T}(M f)$ represents the squared second derivative of the fluence and ensures a smooth fluence.

Two types of constraints are used in this paper: dose-volume and maximum-dose constraints. Each constraint is put into a set $C_{n}$ according to the priority for meeting the constraint level. Constraints in set $C_{0}$ are hard constraints and no relaxation is allowed. The other constraints are soft constraints. Constraints in set $C_{1}$ are the first ones considered for tightening and the last ones to be relaxed, etc. The sets $C_{n}$ represent the constraint priority list. Typical examples are given in tables 1 and 2 .

In the case of constraint violations, involved voxel-coefficients are adapted (sections 2.2.1 and 2.2.2) and a new fluence is calculated by the beam profile optimization. This procedure, designated as coefficients optimization, is repeated until no constraint is violated, or for a maximum number of iterations (section 2.2). 


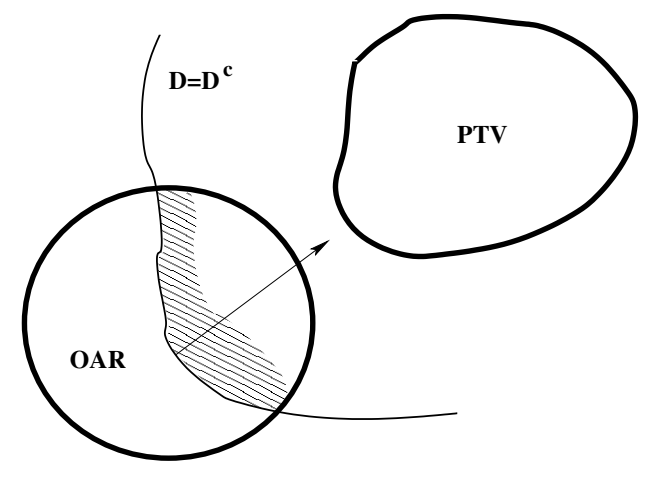

Figure 1. 2D example of voxel selection for iterative voxel-coefficient adaption. $D^{c}$ is the critical dose for the OAR. To reduce the amount of voxels exceeding the critical dose, the value of the voxel-coefficients of the voxels in the shaded area is increased. The result is that the $D^{c}$ isodose line moves closer to the PTV, because dose delivery in the shaded area is penalized.

The four-stage constraint optimization is an iterative multi-criteria optimization mastering the coefficients optimization, based on the constraint priority list. If constraints are too tight and the coefficients optimization cannot find a feasible solution within a fixed number of iterations, constraints are first relaxed. When a feasible solution has been found with relaxed constraints, a process is started to tighten constraints without exceeding any of the other constraints. If none of the constraints can be tightened further, the optimization is terminated (section 2.3).

\subsection{Coefficients optimization}

The coefficients optimization adapts the voxel-coefficient $\boldsymbol{\eta}$ for volumes with violated constraints by increasing the coefficient of one or more voxels. If the minimum dose constraint (mimicked by a dose-volume constraint with $100 \%$ coverage objective) for one or more PTVs is violated, only the voxel-coefficients for these constraints are adapted. Otherwise voxelcoefficients for the other violated constraints are adapted. Note that the coefficient of a voxel can be increased several times in multiple iterations (section 2.3, figure 3 ).

It is also possible to optimize the constraints in prioritized order in this part of the optimization, as was done in Breedveld et al (2007). The drawback is that the constraints will not be relaxed or tightened, which may lead to a suboptimal result or even present an infeasible problem. With a multi-criteria optimization algorithm as presented in section 2.3, also prioritizing the constraints in this part of the optimization is superfluous.

After each adaption of voxel-coefficients, the coefficients optimization performs a beam profile optimization (Breedveld et al 2006) and re-evaluates the constraints. If no constraints have been violated, or after a maximum number of iterations, the coefficients optimization is terminated.

2.2.1. Adaptation of voxel-coefficients for dose-volume constraints. To reduce the highdose volume in the OAR, voxels exceeding the critical dose need to have their doses reduced. Usually, voxels with a high dose are closer to the PTV. Furthermore, it is easier to decrease the dose in voxels with doses close to the critical dose than voxels with a higher dose. Therefore, voxel-coefficients are increased for OAR voxels that just exceed the critical dose $D^{c}$ (figure 1).

The selection of voxels to be adapted is illustrated in more detail in figure 2 for a onedimensional example. The voxels are first sorted in ascending order by dose. For the voxels 

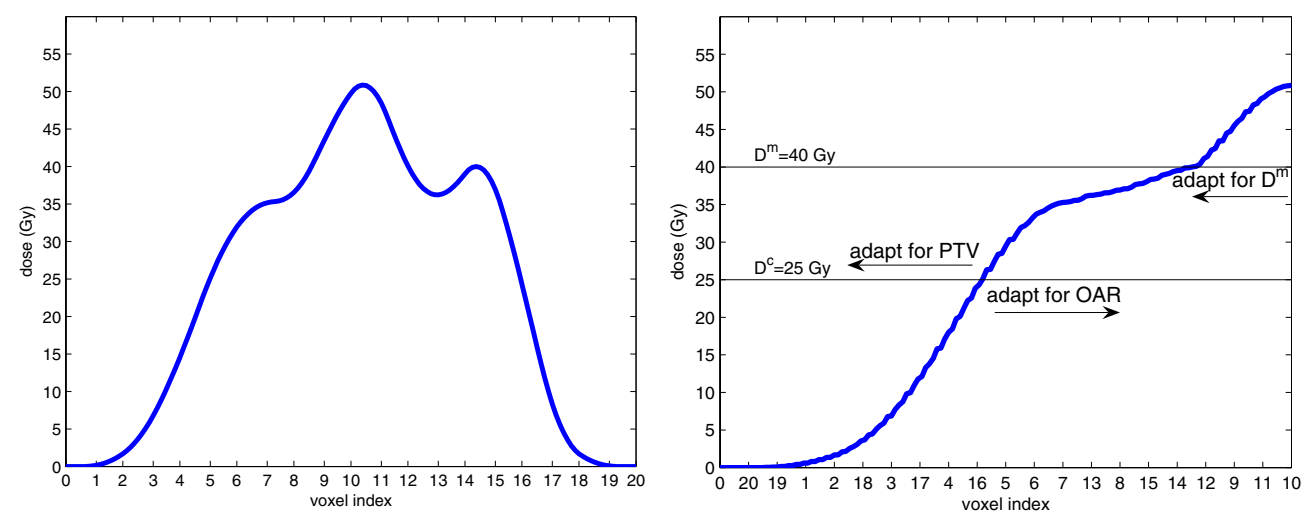

Figure 2. One-dimensional examples of voxel selection for voxel-coefficients adaption. The dose distribution (left) is first sorted in ascending order (right). The first voxel exceeding the critical dose $D^{c}$ of the dose-volume constraint is 16 . For an OAR, the coefficients of voxels $16,5,6, \ldots$ are then adapted. For a PTV voxels $4,17,3, \ldots$ In the case of a dose-maximum constraint $D^{m}$, voxels $10,11,9, \ldots$ are adapted.

exceeding the critical dose $D^{c}$, some voxel-coefficients are increased. When applied to the PTV, the voxels are adapted from the high-dose region just below $D^{c}$ to the low-dose region. Bortfeld et al (1997) used the same idea applied to a penalty function. In the approach of Cotrutz and Xing (2002), dosimetrists have to manually select areas in the DVH that need improvement.

2.2.2. Adaptation of voxel-coefficients for maximum-dose constraints. A similar technique is applied to suppress high-dose regions: the coefficients for a subset of the voxels receiving a dose larger than $D^{m}$ are adapted, starting with the voxels receiving the highest doses (see also figure 2).

\subsection{Constraint optimization}

The coefficients optimization may be used to generate a solution that meets a set of constraints, but it fails to come up with an alternative if the constraints are too tight. On the other hand, if the constraints are too loose, the solution found is sub-optimal, unless the constraints are set just tight enough.

In our multi-criteria approach, constraint optimization based on the initial constraints and the constraint priority list is used to generate a plan with the property that improving a single constraint is only possible if at least one other constraint is violated.

The constraint optimization is a four-stage process (figure 3). Each stage calls the coefficients optimization with a maximum number of iterations $\left(N_{1}, \ldots, N_{4}\right.$ for each stage). The coefficients optimization is an evolutionary algorithm, i.e., the voxel-coefficient vector is updated in each iteration. This property is also used in the constraint optimization because the solution of a slightly different constraint set (i.e. one constraint is more tight) lies in the proximity of the current solution. This allows a fast search through the constraint space because it is not required to start from scratch in each iteration of the constraint optimization.

2.3.1. Stage 1. Before the first stage starts, a single optimization is done of the quadratic objective function with voxel weights 1 for the PTV(s) and 0 for all OAR. This results in an 


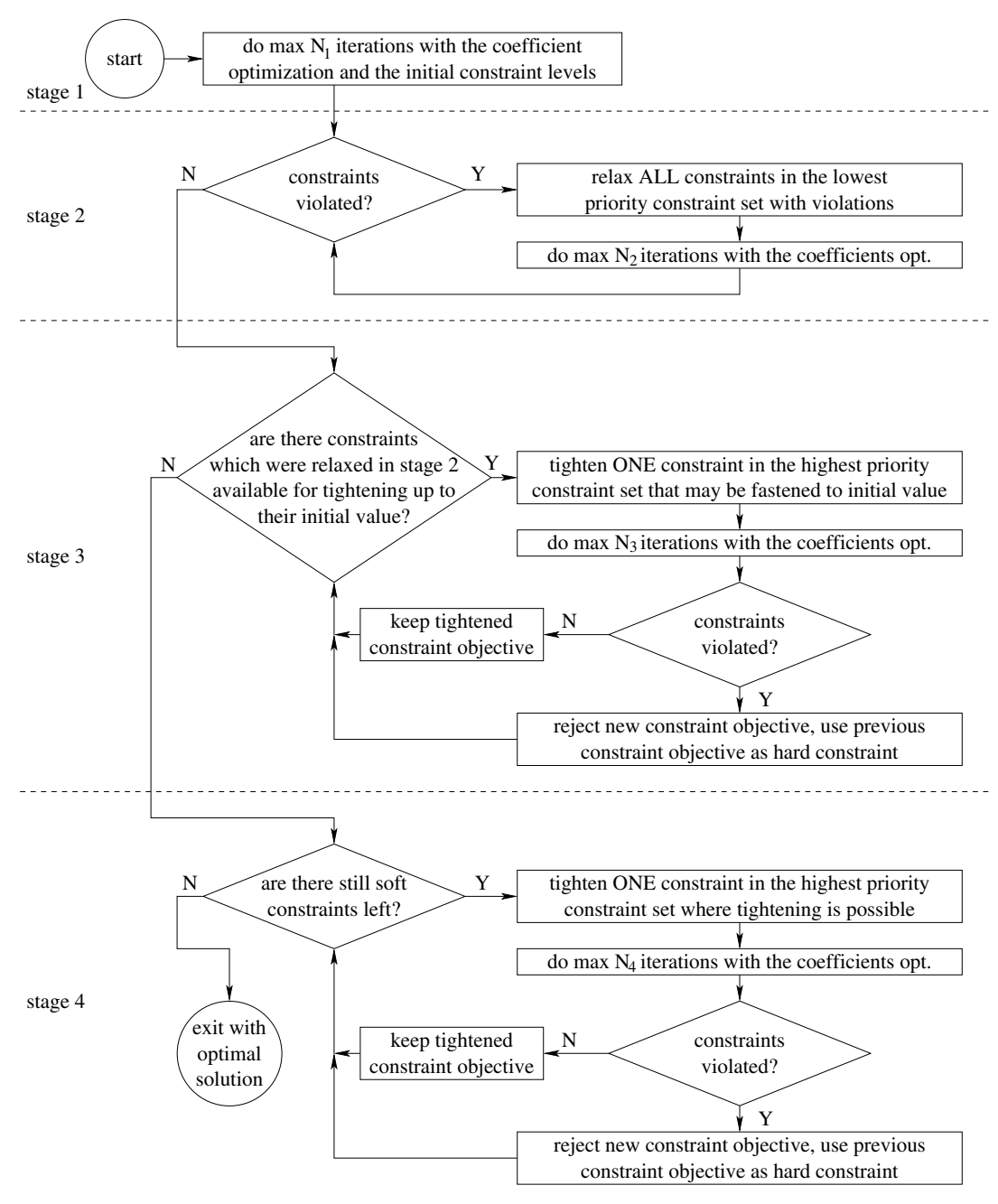

Figure 3. Flow diagram of the four-stage constraint optimization. The first stage populates the coefficient vector with problematic voxels. If soft constraints cannot be met, they are relaxed in the second stage. The third stage tries to undo this relaxation, by tightening relaxed constraints up to the initial constraint levels. Finally, the fourth stage tightens soft constraints until no further tightening of any of them is possible.

initial dose distribution. Then the first stage is started and a maximum of $N_{1}$ iterations are done with the coefficients optimization, subjected to the initial constraints. This populates the voxel-coefficient $\boldsymbol{\eta}$ with 'problematic' voxels.

2.3.2. Stage 2. If a plan satisfying all constraints is not found in the first stage, violated soft constraints are relaxed in the second stage. The constraints in the set $C_{n}$ with the lowest priority are selected for relaxation first, if there are one or more constraints violated in that set. A constraint is relaxed by setting it to the upper rounding of the current solution, so if the initial objective is $40 \%$ and the current solution is $43.21 \%$, the new objective for the constraint is set to $44 \%$. Then $N_{2}$ iterations with the coefficients optimization are performed to search for a solution. These iterations are also done if no constraints are suitable for relaxation (e.g. 
only the hard constraints are not satisfied). The second stage is repeated until a plan is found that fulfils all hard constraints and all (relaxed) soft constraints. Here it is assumed that there are no conflicting hard constraints.

2.3.3. Stages 3 and 4 . In these stages, the stage 2 plan is further optimized by tightening soft constraints one by one, so a plan is generated such that further tightening of one constraint will always lead to a violation of at least one other. In stage 3, attempts are made to tighten constraints that were relaxed in stage 2 with the ultimate goal to reach the initial values, i.e., to undo the relaxations. The first constraints to be considered are in the highest priority constraint set $\left(C_{1}\right)$. If after a maximum of $N_{3}$ iterations of the coefficients optimization all constraints are met, including the tightened constraint, the tightened objective for the latter is kept. Otherwise, the constraint is reset to its previous objective, the steps of the coefficients optimization are undone, and the constraint no longer participates in the constraint optimization, and becomes virtually a hard constraint for the rest of the constraint optimization. In this procedure, a constraint is tightened by rounding off to the lower integer, so if the current solution has a value of $43.21 \%$, the new objective for this constraint will be $43 \%$. For dose-volume constraints, this results in minimizing the number of voxels receiving more than the critical dose (Halabi et al (2006) also uses this concept in a different problem definition).

As mentioned, stage 3 aims at undoing the relaxations done in stage 2 as much as possible, with the final goal to generate a plan that fulfils to all initial constraints. Stage 4 takes this a step further. In a similar procedure as for stage 3, constraints are tightened further with an attempt to make them more strict than the initial objectives. Important to note is that in stages 3 and 4 tightening of important constraints may result in less favourable results for less important constraints. However, the tightening will never result in exceeding any of the (current) constraint levels.

\subsection{Calculations}

The algorithm used to calculate the dose deposition matrix $H$ is from Storchi and Woudstra (1996) and uses inhomogeneity corrections for air cavities and a scatter radius of $3 \mathrm{~cm}$. The Storchi and Woudstra algorithm for dose calculation is comparable to the one used in the CadPlan treatment planning system. The pixel grid size is $5 \times 10 \mathrm{~mm}^{2}$ and the voxel grid size is $4 \times 4 \times 5 \mathrm{~mm}^{3}$ for all patients. The numbers of iterations performed with the coefficients optimization are $\left(N_{1}, N_{2}, N_{3}, N_{4}\right)=(50,1,30,20)$. Computations were performed in Matlab 7 on a $2.4 \mathrm{GHz}$ Intel Core 2 workstation running Gentoo Linux.

\section{Results}

The proposed method for multi-criteria inverse planning has been applied to patients suffering from rectum cancer and oropharynx cancer, and to a complicated head and neck case. For rectum and oropharynx cancer it has been demonstrated that the proposed approach can be used to generate class solutions for patients with the same tumour type. To find a constraint list which performs well as a class solution, different lists were tried and their results and performance were compared for four patients. The final constraint list was used on four other rectum patients to test the general performance. All generated plans are compared to the corresponding clinically applied plans made in CadPlan (referred to as the clinical plan). Here results are presented for the rectum cases and for the complex head and neck case. 


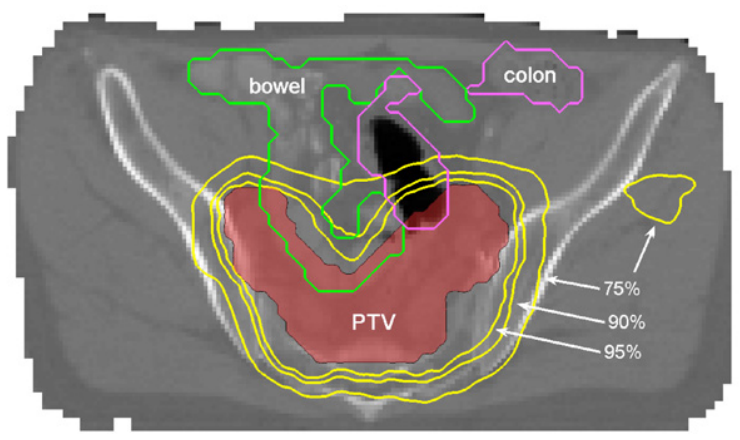

Figure 4. Volumes and dose distribution for the third rectum case.

Table 1. Constraints and priorities for treatment of rectal cancer. Dose-volume constraints are indicated by DV.

\begin{tabular}{clllll}
\hline No & Volume & $\begin{array}{l}\text { Constraint } \\
\text { type }\end{array}$ & $\begin{array}{l}\text { Critical } \\
\text { dose }\end{array}$ & $\begin{array}{l}\text { Constraint } \\
\text { set }\end{array}$ \\
\hline 1 & PTV & DV & $42.42 \mathrm{~Gy}$ & $\begin{array}{l}100 \% \\
47.78 \mathrm{~Gy}\end{array}$ & 0 \\
2 & PTV & Max & & $47.78 \mathrm{~Gy}$ & 0 \\
3 & Body & Max & & $20 \%$ & 1 \\
4 & Bowel & DV & $35 \mathrm{~Gy}$ & $40 \%$ & 2 \\
5 & Bladder & DV & $40 \mathrm{~Gy}$ & $20 \%$ & 2 \\
6 & Colon & DV & $40 \mathrm{~Gy}$ & $50 \%$ & 3 \\
7 & Bowel & DV & $20 \mathrm{~Gy}$ & $75 \%$ & 3 \\
8 & Bladder & DV & $20 \mathrm{~Gy}$ & $30 \%$ & 3 \\
9 & Colon & DV & $20 \mathrm{~Gy}$ & $30 \%$ & 4 \\
10 & Body & DV & $30 \mathrm{~Gy}$ & $40 \%$ \\
\hline
\end{tabular}

\subsection{Rectum}

All patients were to be treated with a prescribed dose of $44.65 \mathrm{~Gy}$ (19 fractions of $2.35 \mathrm{~Gy}$ ). The constraint list (table 1) has been tuned to produce desired results on four patients by defining the dose-volume points and the subdivision into constraint sets. The importance factors for the PTV, bowel, bladder, colon and body have been chosen to be $100,10,5,5$ and 1, respectively. To make a fair comparison, the beam directions and energies are chosen identical to the ones used in the clinical plan: five beams of $18 \mathrm{MV}$ around 85, 155, 180, 205 and $275^{\circ}$, where sometimes the beam for $180^{\circ}$ was chosen $6 \mathrm{MV}$. The performance of this protocol was verified for four other patients.

For patient 3 , the resulting dose distribution is displayed in figure 4. It shows that the algorithm is capable of generating highly conformal dose distributions. The dose-volume histograms in figure 5 show that our algorithm for multi-criteria optimization does not only improve OAR dose volume histograms, but also the PTV dose homogeneity (avoidance of hot spots).

\subsection{Complex head and neck case}

An extensive nasopharynx carcinoma case was selected to investigate the value of the developed algorithm when a large number of structures are involved. The PTV starts halfway down the eyes, branches into two neck regions (right neck is positive) and ends in front of the lung tops 

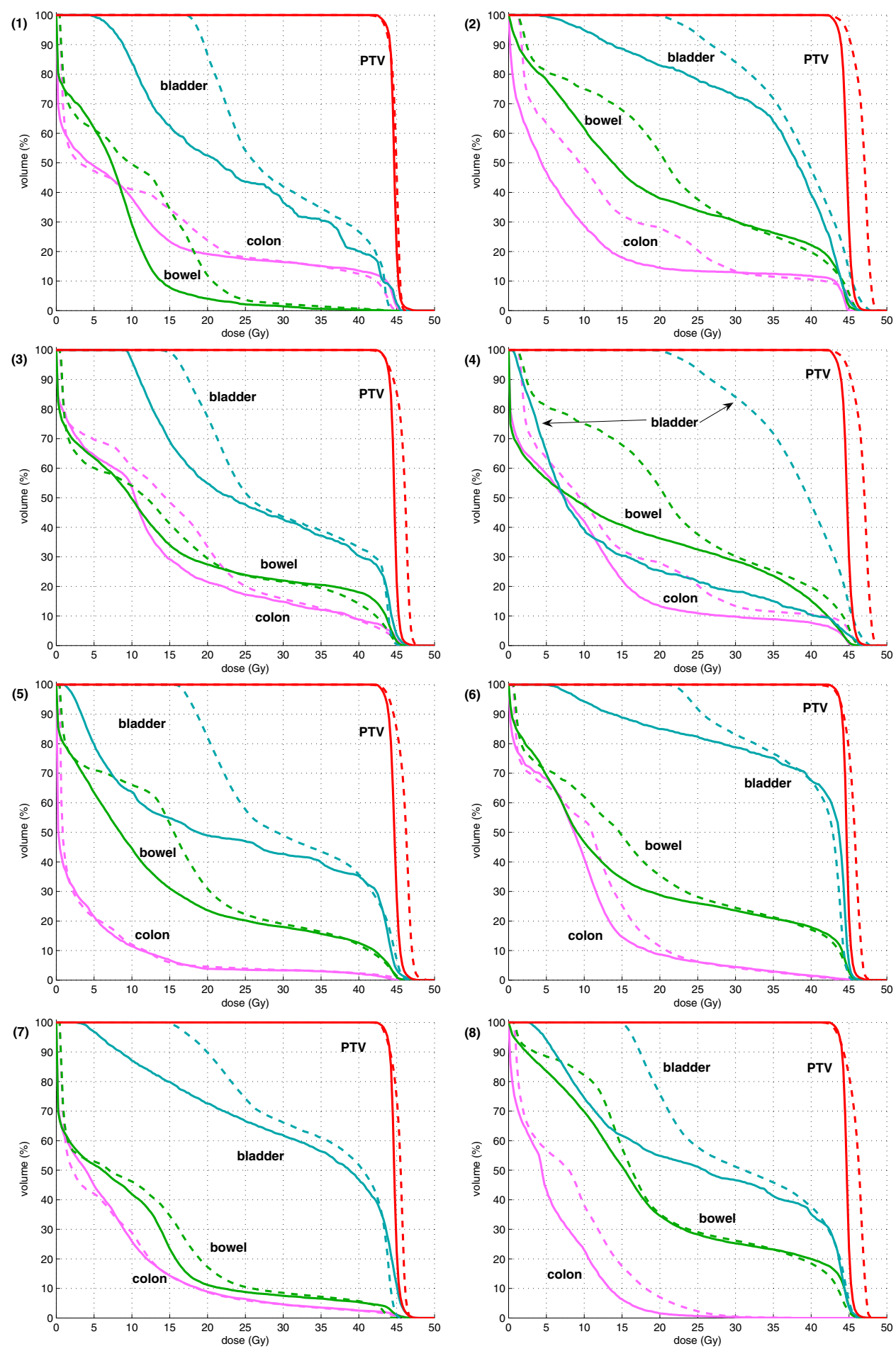

Figure 5. Dose-volume histograms for the eight rectum patients. Histograms are presented for the clinical IMRT plan (dashed lines) and for the plan generated with the developed method for automated multi-criteria optimization. 
Table 2. Constraints, priorities and results for the complex head and neck case. PTV46 is the collection of the primary tumour, positive neck and elective nodes. PTV70 is the primary tumour and the positive neck.

\begin{tabular}{|c|c|c|c|c|c|c|c|c|}
\hline No & Volume & $\begin{array}{l}\text { Constraint } \\
\text { type }\end{array}$ & $\begin{array}{l}\text { Critical } \\
\text { dose }\end{array}$ & Objective & $\begin{array}{l}\text { Realized } \\
\text { objective }\end{array}$ & $\begin{array}{l}\text { Mean } \\
\text { dose }\end{array}$ & $\begin{array}{l}\text { Clinical } \\
\text { realized } \\
\text { objective }\end{array}$ & $\begin{array}{l}\text { Constraint } \\
\text { set }\end{array}$ \\
\hline 1 & PTV46 & DV & $43.7 \mathrm{~Gy}$ & $100 \%$ & $100.0 \%$ & & $96.5 \%$ & 0 \\
\hline 2 & Sella & Max & & $55 \mathrm{~Gy}$ & $55.0 \mathrm{~Gy}$ & & $52.6 \mathrm{~Gy}$ & 0 \\
\hline 3 & Myelum & $\operatorname{Max}$ & & $45 \mathrm{~Gy}$ & $44.8 \mathrm{~Gy}$ & & $51.6 \mathrm{~Gy}$ & 0 \\
\hline 4 & Optic chiasm & Max & & $50 \mathrm{~Gy}$ & $50.0 \mathrm{~Gy}$ & & $52.5 \mathrm{~Gy}$ & 0 \\
\hline 5 & Optical nerve (L) & $\operatorname{Max}$ & & $55 \mathrm{~Gy}$ & $55.0 \mathrm{~Gy}$ & & $47.3 \mathrm{~Gy}$ & 0 \\
\hline 6 & Optical nerve $(\mathrm{R})$ & $\operatorname{Max}$ & & $55 \mathrm{~Gy}$ & $52.0 \mathrm{~Gy}$ & & $48.5 \mathrm{~Gy}$ & 0 \\
\hline 7 & Eye (L) & Max & & 35 Gy & $35.0 \mathrm{~Gy}$ & & $42.9 \mathrm{~Gy}$ & 0 \\
\hline 8 & Eye $(\mathrm{R})$ & Max & & $35 \mathrm{~Gy}$ & $35.0 \mathrm{~Gy}$ & & $42.5 \mathrm{~Gy}$ & 0 \\
\hline 9 & PTV70 & Max & & $74.9 \mathrm{~Gy}$ & $74.8 \mathrm{~Gy}$ & & $78.9 \mathrm{~Gy}$ & 0 \\
\hline 10 & PTV70 & DV & $66.5 \mathrm{~Gy}$ & $100 \%$ & $93.2 \%$ & & $89.1 \%$ & 1 \\
\hline 11 & Brainstem & DV & $55 \mathrm{~Gy}$ & $0 \%$ & $0.0 \%$ & & $1.2 \%$ & 2 \\
\hline 12 & Pons & DV & $55 \mathrm{~Gy}$ & $0 \%$ & $0.0 \%$ & & $2.9 \%$ & 2 \\
\hline 13 & Parotid (L) & DV & $26 \mathrm{~Gy}$ & $50 \%$ & $46.1 \%$ & $27.0 \mathrm{~Gy}$ & $39.2 \%$ & 3 \\
\hline 14 & Oral cavity & DV & 26 Gy & $50 \%$ & $48.0 \%$ & $30.2 \mathrm{~Gy}$ & $100.0 \%$ & 4 \\
\hline 15 & Pharynx/trachea & DV & $40 \mathrm{~Gy}$ & $40 \%$ & $24.2 \%$ & $34.5 \mathrm{~Gy}$ & $44.3 \%$ & 4 \\
\hline 16 & Lung tops & DV & $18 \mathrm{~Gy}$ & $20 \%$ & $6.5 \%$ & $6.5 \mathrm{~Gy}$ & $20.0 \%$ & 4 \\
\hline 17 & Body & DV & $40 \mathrm{~Gy}$ & $90 \%$ & $18.9 \%$ & & $\mathrm{~N} / \mathrm{A}^{\mathrm{a}}$ & 5 \\
\hline
\end{tabular}

a Definition of (external) body contour differs between CadPlan and our algorithm.

(level IV). The PTV length is approximately $28 \mathrm{~cm}$ and the total volume is $800 \mathrm{cc}$. The optical nerves and optic chiasm do partially overlap with the primary tumour/positive neck.

The constraint list used is shown in table 2, containing 17 constraints for the 16 volumes involved. The whole PTV should receive at least $95 \%$ of the prescribed dose of $46 \mathrm{~Gy}$ (43.7 Gy, hard constraint). The volume of the gross tumour (PTV70) should receive at least 66.5 Gy. However, because at the same time, the organs at risk have to be protected by hard (maximum-dose) constraints, the criterion to deliver 66.5 Gy to the PTV70 has been relaxed into a soft constraint with priority 1.

The volume-wide importance factors are 100 for the $70 \mathrm{~Gy}$ PTV, 50 for the $46 \mathrm{~Gy}$ PTV, 10 for the sella, optic chiasm, optical nerves and parotid, 5 for the brainstem, pons, eyes, oral cavity and pharynx/trachea and 1 for the myelum, lung tops and body. The optimized plan is compared to the clinical plan, which took experienced planners two weeks to achieve with 16 IMRT and wedged beams. Our setup uses nine equi-angular 6 MV beams.

The results are presented in table 2 and figure 6 . Table 2 shows that using the developed multi-criteria approach all hard constraints could be met. In contrast to the clinical plan, with the multi-criteria approach the full PTV46 could be irradiated with the critical dose. Because of this improved tumour coverage, the mean dose in the parotid is slightly higher than in the clinical plan (27.0 Gy mean dose instead of 25.6 Gy in the clinical plan). The dose in the oral cavity is significantly lower than in the clinical plan: the mean dose reduces from 55.5 Gy to 30.2 Gy.

Figure 7 shows the convergence of some dose-volume constraints. The first stage ends after 50 iterations without finding a plan meeting all hard constraints. This requires another 240 iterations (stage 2). Note the decrease in coverage of the 70 Gy PTV which grows to $100 \%$ in the first iterations, but decreases to $93 \%$ afterwards. The third stage, trying to undo the constraint relaxations of stage 2, starts after 290 iterations (indicated by (a)). 

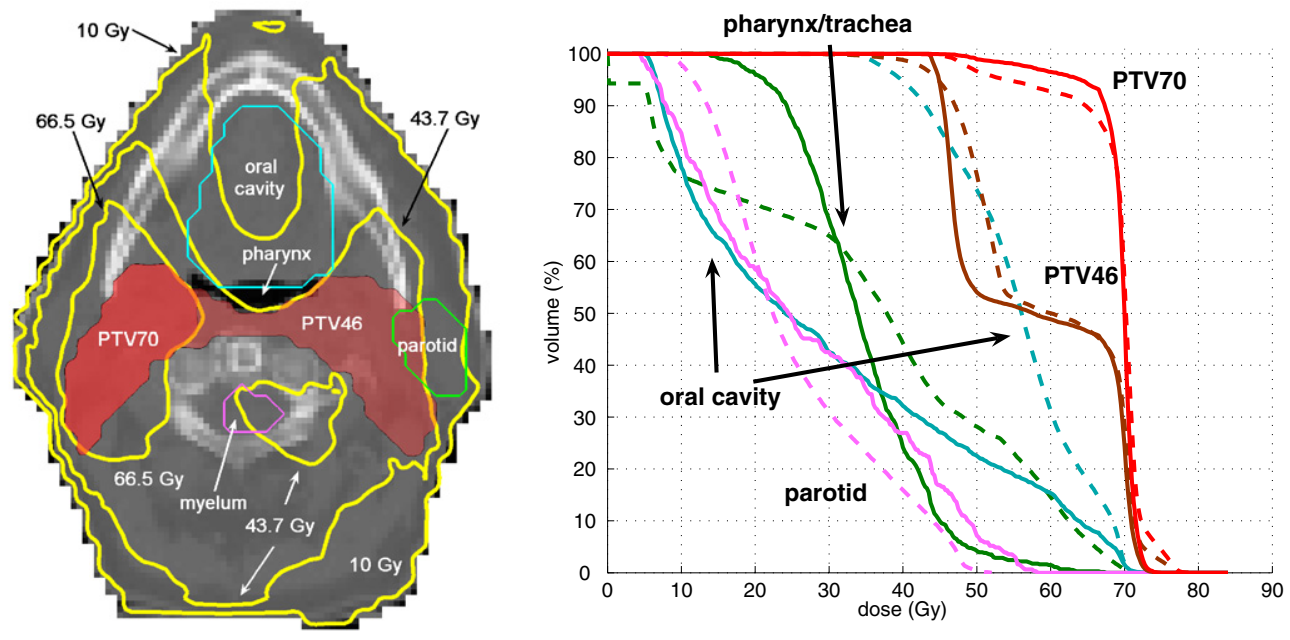

Figure 6. The left figure shows a CT slice with isodose lines for doses prescribed to PTV70 (66.5 Gy), PTV46 (43.7 Gy) and the $10 \mathrm{~Gy}$ isodose line. The right figure shows the dose-volume histograms for some interesting volumes, where the dashed lines indicate the results of the clinical plan.

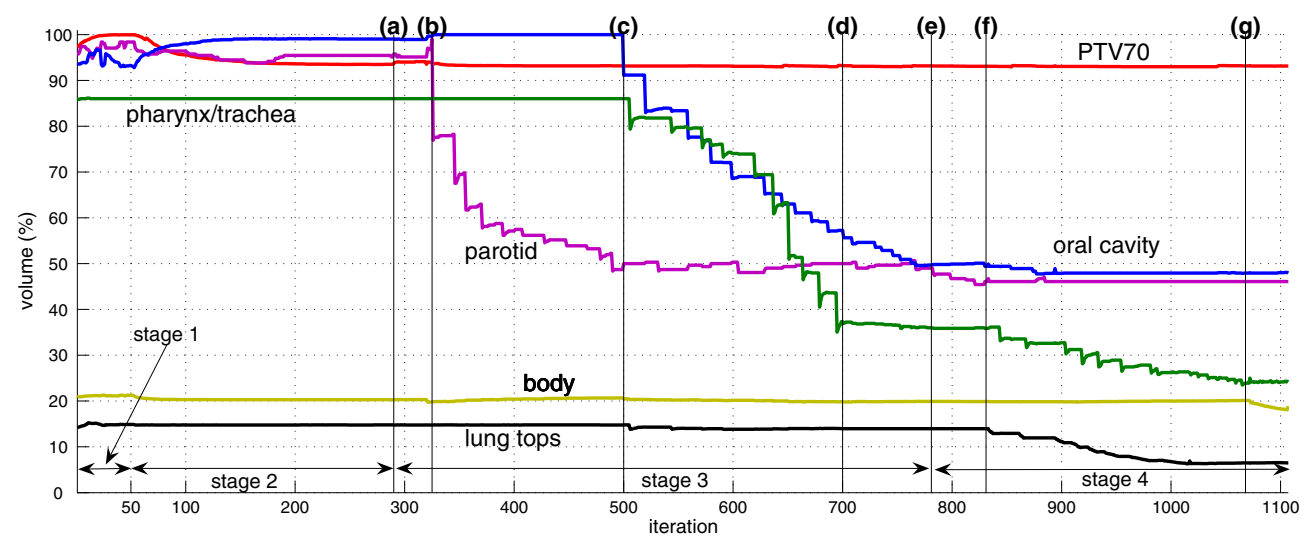

Figure 7. Convergence of the dose-volume constraints. The letters (a)-(g) are explained in the text.

Between (a) and (b), the algorithm minimizes the dose-volume constraints for the brainstem and pons (not shown in figure). After their initial objective of $0 \%$ overdose is met, the dose-volume constraint for the parotid is tightened (b) until its initial objective of $50 \%$ (c). The oral cavity, pharynx/trachea and lung tops are all in constraint set 4 . Since the dosevolume constraint for the lung tops is not violated, only the constraints for the oral cavity and pharynx/trachea are tightened (in turn) until the initial objectives are met, (d) and (e) for the pharynx/trachea and the oral cavity, respectively. Now that all initial constraints have been met (as far as the hard constraints allow), stage 4 is initiated. Firstly, the constraint for the parotid is tightened which reaches its minimum value after a small reduction (f). Then the oral cavity, pharynx/trachea, and also the lung tops are considered. When no tightening is possible anymore, the algorithm tightens the constraints in the last set, containing only the body/unspecified tissue (g). 

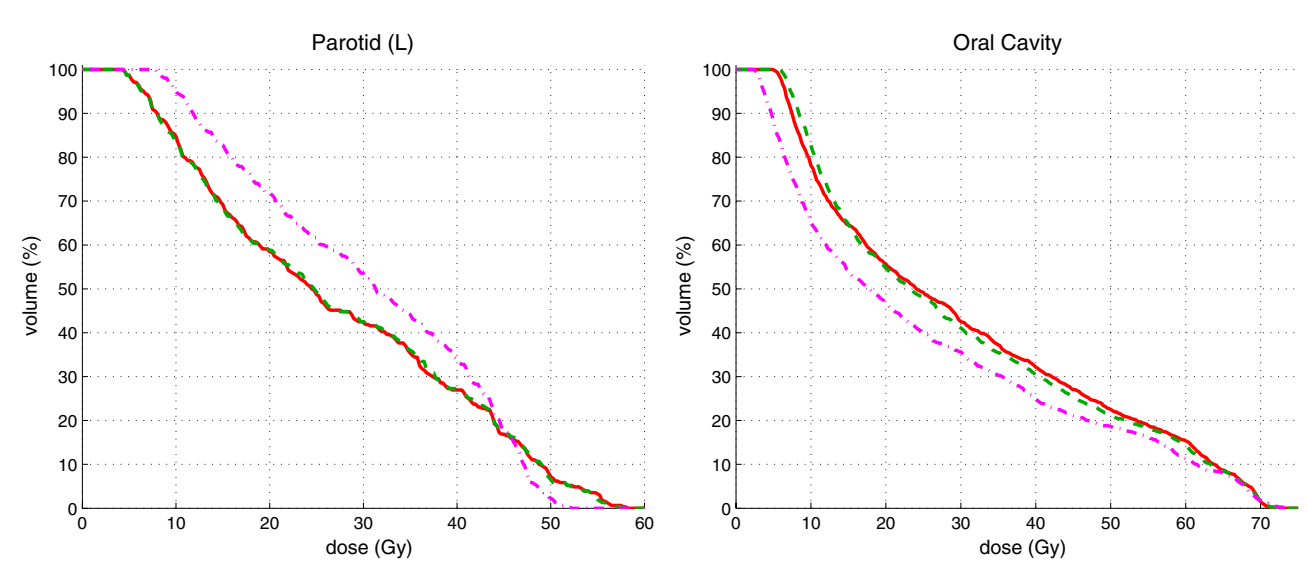

Figure 8. Dose-volume histograms for the parotid (left) and the oral cavity (right). The solid line is the original solution. The dashed line is the solution when their priorities are interchanged and the dash-dotted line when the imposed objective for the parotid is increased to $70 \%$ as well.

A direct comparison between the clinical plan and the automated plan is not possible for this case. This case is too extreme to be handled efficiently by CadPlan. There are 16 volumes where CadPlan can only handle 10 at a time in an optimization, so it was not possible to minimize all constraints simultaneously. Further, the 46 Gy plan and the boost plan were planned separately and combined later. The beam setup differed as well (16 IMRT and wedged beams in the clinical plan compared to 9 in our plan). CadPlan also uses a weighted sum function for optimization. A weighted sum function is inefficient in making sharp trade-offs (e.g. 100\% tumour coverage and minimizing dose in a volume close to the PTV) because it mixes objectives with constraints. These reasons explain why it was possible to improve the mean dose in the oral cavity by $15 \mathrm{~Gy}$.

\subsection{Constraint list sensitivity}

The final result depends on the initial objectives of the constraints and the constraint set they are assigned to. In this section we describe how the outcome of the algorithm reacts to the chosen criteria. Results are shown for the parotid and oral cavity of the complex head and neck case in figure 8 .

When only the priorities for the parotid and oral cavity ( 3 and 4 , respectively) are interchanged, the final dose-volume histograms are almost unchanged. When in the third stage of the constraint optimization the imposed objective of $50 \%$ is reached for both the parotid and oral cavity, the constraint on the parotid does not leave much space to improve the dose to the oral cavity. If the imposed objective for the parotid is increased (relaxed) to $70 \%$, the oral cavity is much better spared.

When the realized objectives of the final solution were used as imposed objectives for a new optimization, the resulting dose distributions were virtually identical to the previous distributions. This is an indication that the algorithm is robust, because when different starting positions (constraint lists) are used, the same result is obtained. None of the cases could be significantly improved, which demonstrates the optimality of the constraints.

But interchanging priorities or relaxing imposed objectives does not guarantee that another solution can be obtained, when the minimum-dose for the PTV is kept as a hard constraint. The physiology of the patient must also allow the desired trade-offs. For example, for the rectum 
patients, interchanging the priorities for the bowel and the colon, or changing the imposed objectives, does not permit much difference in the final solution: this is because the volumes are physically correlated. The placement and the number of beams used play important roles as well.

Another parameter of influence is the volume-wide importance factor for each volume. Because the voxels belonging to a certain volume are multiplied by their volume-wide importance factor, the choice of the volume-wide importance factor plays a minor role in the voxel-dependent approach, and mainly influences the number of iterations required to obtain the final solution, because it influences the impact of an adapted voxel-weight. If a volume has a large volume-wide importance factor, the effect of an adapted voxel is large as well, and the coefficients optimization needs more iterations to obtain a solution fulfilling all constraints. A rule of thumb for the volume-wide importance factors is (relative) 100 for the PTV, 50 for elective glands (PTV), 5-10 for important OARs and 1 for other OARs.

\section{Discussion and conclusions}

We have developed an algorithm for multi-criteria inverse planning for IMRT. Input is a constraint priority list, to be provided by the radiation oncologist. In this list, constraints are ranked in groups, according to the priority for meeting the constraint levels. Constraints with the highest priority are considered hard constraints that necessarily have to be met, i.e., they cannot be relaxed in order to improve compliance with other constraints. In an iterative procedure, using an algorithm for optimization of voxel-dependent importance factors for the various dose-volume and maximum-dose constraints, soft constraints are optimized so that the final list of constraints has the property that if one constraint is tightened further, it would lead to a violation of at least one other constraint.

For the final constraints, this follows the definition of Pareto optimality. Our four-stage constraint optimization can be seen as an implementation of the $\epsilon$-constraint method (Haimes et al 1971, Hoffmann et al 2006, Jee et al 2007). This method minimizes a set of objectives by minimizing one objective at a time, while keeping the others constrained. The minimum value for that objective is used as a constraint in the next iteration(s) where another objective is minimized.

In clinical practice, the automated approach is able to reduce the workload because it is able to come up with satisfactory plans for routine cases, where it is possible to determine the trade-offs a priori. Of course, there will always remain cases where an automated approach is not satisfactory. Different constraint lists have to be tried, as well as possibly different beam setups, requiring a rerun of the optimization. For these special cases, presenting the radiation oncologist with a set of (Pareto optimal) plans might be more efficient. Many authors have looked into this (e.g. Küfer et al 2005, Craft et al 2005, 2006, Halabi et al 2006, Hoffmann et al 2006). Our multi-criteria algorithm is not capable of producing multiple solutions because it was designed to provide only one solution which is within, or closest to the initial constraints, and may be even obeying tighter constraints than initially prescribed.

For rectal and oropharynx cancer patients, the developed multi-criteria approach was used for fully automated generation of class solutions. For the rectum patients, the class solutions were superior to the clinical plans, both regarding obtained tumour dose distributions, and with respect to OAR sparing. For the oropharynx patients plans were similar (data not shown), but the automated procedure was faster and required less workload.

For violated constraints, voxel-weights are automatically adapted. Cotrutz and Xing (2002) use a semi-automatic approach where the dosimetrist manually has to select the part of the DVH where improvement is desired. Based on the selected areas, relevant voxel-dependent 
Table 3. (Average) optimization statistics.

\begin{tabular}{llclc}
\hline Case & Time & Iterations & Beamlets & Time per iteration \\
\hline Rectum patients & $47(\mathrm{~m})$ & 345 & 2163 & $8.1(\mathrm{~s})$ \\
Complex head and neck case & $38(\mathrm{~h})$ & 1107 & 5293 & $125.2(\mathrm{~s})$ \\
\hline
\end{tabular}

importance factors are then adjusted, followed by a new optimization. In this paper we have proposed a scheme for automatic adjustments of voxel-dependent importance factors, also based on DVH. In a later work, Yang and Xing (2004) propose a different voxel weight update scheme for automated adaption.

Some optimization statistics are given in table 3. The average optimization time of $47 \mathrm{~min}$ for rectal cancer is acceptable considering that the final result is a Pareto optimal solution and there is no human intervention. The optimization time is related to the number of beamlets and number of soft constraints. The complex, extended head and neck case with 17 constraints for the 16 involved organs required almost $40 \mathrm{~h}$ of calculation time. The obtained plan is superior to the corresponding clinical plan. Generation of the latter took two weeks of work of a highly experienced dosimetrist.

\section{Acknowledgments}

The authors want to thank Peter Voet for his help on defining treatment goals for the patients, and for reviewing and discussing the results. The authors would also like to thank the referees for their critical and stimulating comments.

\section{References}

Bortfeld T, Burkelbach J, Boesecke R and Schlegel W 1990 Methods of image reconstruction from projections applied to conformation radiotherapy Phys. Med. Biol. 35 1423-34

Bortfeld T, Stein J and Preiser K 1997 Clinically relevant intensity modulation optimization using physical criteria Proc. XII Int. Conf. Use of Computers in Radiation Therapy (Salt Lake City, UT)

Brahme A 1995 Treatment optimization using physical and radiobiological objective functions Radiation Therapy Physics ed A R Smith (Berlin: Springer) pp 209-46

Breedveld S, Storchi P R M, Keijzer M and Heijmen B J M 2006 Fast, multiple optimizations of quadratic dose objective functions in IMRT Phys. Med. Biol. 51 3569-79

Breedveld S, Storchi P R M, Keijzer M and Heijmen B J M 2007 Automated adaption of voxel-dependent importance factors in inverse planning Proc. XV Int. Conf. Use of Computers in Radiation Therapy (Toronto, Canada) vol 1, pp 232-6

Cotrutz C, Lahanas M, Kappas C and Baltas D 2001 A multiobjective gradient-based dose optimization algorithm for external beam conformal radiotherapy Phys. Med. Biol. 46 2161-75

Cotrutz C and Xing L 2002 Using voxel-based importance factors for interactive DVH-based dose optimization Phys. Med. Biol. 47 1659-69

Cotrutz C and Xing L 2003 IMRT dose shaping with regionally variable penalty scheme Med. Phys. 30 544-51

Craft D, Halabi T and Bortfeld T 2005 Exploration of tradeoffs in intensity-modulated radiotherapy Phys. Med. Biol. $505857-68$

Craft D L, Halabi T F, Shih H A and Bortfeld T R 2006 Approximating convex Pareto surfaces in multiobjective radiotherapy planning Med. Phys 33 3399-407

Haimes Y Y, Lasdon L S and Wismer D A 1971 On a bicriterion formulation of the problems of integrated system identification and system optimization IEEE Trans. Syst. Man Cybern. $1296-7$

Halabi T, Craft D and Bortfeld T 2006 Dose-volume objectives in multi-criteria optimization Phys. Med. Biol. 51 3809-18

Hoffmann A L, Siem A Y D, den Hertog D, Kaanders J H A M and Huizenga H 2006 Derivative-free generation and interpolation of convex Pareto optimal IMRT plans Phys. Med. Biol. 51 6349-69 
Jee K-W, McShan D L and Fraass B A 2007 Lexicographic ordering: intuitive multicriteria optimization for IMRT Phys. Med. Biol. 52 1845-61

Küfer K-H, Monz M, Scherrer A, Süss P, Alonso F, Sultan A S A, Bortfeld T, Craft D and Thieke C 2005 Multicriteria optimization in intensity modulated radiotherapy planning Berichte des Fraunhofer ITWM vol 77 (Kaiserslautern, Germany: Fraunhofer Institut Techno- und Wirtschaftsmathematik)

Lahanas M, Schreibmann E and Baltas D 2003 Multiobjective inverse planning for intensity modulated radiotherapy with constraint-free gradient-based optimization algorithms Phys. Med. Biol. 48 2843-71

Scherrer A, Küfer K-H, Bortfeld T, Monz M and Alonso F 2005 IMRT planning on adaptive volume structures-a decisive reduction in computational complexity Phys. Med. Biol. 50 2033-53

Shou Z, Yang Y, Cotrutz C, Levy D and Xing L 2005 Quantitation of the a priori dosimetric capabilities of spatial points in inverse planning and its significant implication in defining IMRT solution space Phys. Med. Biol. 50 1469-82

Spirou S V and Chui C S 1998 A gradient inverse planning algorithm with dose-volume constraints Med. Phys. 25 321-33

Storchi P R M and Woudstra E 1996 Calculation of the absorbed dose distribution due to irregularly shaped photon beams using pencil beams kernels derived from basic beam data Phys. Med. Biol. 41 637-56

Wilkens J J, Alaly J R, Zakarian K, Thorstad W L and Deasy J O 2007 IMRT treatment planning based on prioritizing prescription goals Phys. Med. Biol. 52 1675-92

Wu Q and Mohan R 2000 Algorithms and functionality of an intensity modulated radiotherapy optimization system Med. Phys. 27 701-11

Wu C, Olivera G H, Jeraj R, Keller H and Mackie T R 2003 Treatment plan modification using voxel-based weighting factors/dose prescription Phys. Med. Biol. 48 2479-91

Yang Y and Xing L 2004 Inverse treatment planning with adaptively evolving voxel-dependent penalty scheme $M e d$. Phys. 31 2839-44 